\title{
$\angle S$ Research Square \\ Preferences and health utilities related to disease and treatment features for patients with hemophilia A in Canada
}

Karissa Johnston ( $\square$ kjohnston@broadstreetheor.com )

Broadstreet Health Economics and Outcomes Research https://orcid.org/0000-0003-4570-9972 Jayson Stoffman

Department of Pediatrics, Max Rady College of Medicine, University of Manitoba

\section{Alexis Mickle}

Broadstreet Health Economics and Outcomes Research

\section{Robert Klaassen}

Department of Pediatrics, Children's Hospital of Eastern Ontario Research Institute, University of Ottawa

\section{Demitri Diles}

Hoffmann-La Roche Limited

Shade Olatunde

Hoffmann-La Roche Limited

\section{Lina Eliasson}

Sprout Behavior Change Ltd.

\section{Roxana Bahar}

Sprout Behavior Change Ltd.

\section{Research}

Keywords: Canadian societal perspective, health-related quality of life, utilities, hemophilia A

Posted Date: December 23rd, 2020

DOI: https://doi.org/10.21203/rs.3.rs-133513/v1

License: (c) (i) This work is licensed under a Creative Commons Attribution 4.0 International License.

Read Full License 


\section{Abstract}

Background: Hemophilia A is caused by a mutation of clotting factor genes resulting in a deficiency of factor VIII (FVIII). Current treatments for hemophilia A in Canada include on-demand treatment as bleeds occur and regular intravenous prophylactic FVIII infusions. The subcutaneous therapy emicizumab was recently approved for the treatment of hemophilia A. The objective of this study was to estimate utility values associated with hemophilia A health and treatment states from a Canadian societal perspective, including preferences related to treatment efficacy as well as frequency and route of administration.

Methods: A vignette-based time trade-off (TTO) utilities elicitation was undertaken to compare population preferences for 6 hemophilia health states describing prophylactic and on-demand treatment, with varying bleed rates and frequency of treatment administration. Health state definitions were informed by clinical experience, HAVEN3 results regarding bleed frequency, and supplemented with qualitative interviews of hemophilia patients and caregivers $(n=10)$.

Results: TTO interviews were conducted with 82 general population respondents. Mean utilities [95\% $\mathrm{Cl}]$ were highest for subcutaneous prophylaxis $(0.90$ [0.87-0.93]), followed by intravenous prophylaxis $(0.81$ [0.78-0.85]), with on-demand having the lowest utility (0.70 [0.65-0.76]). In regression analysis, subcutaneous treatment health states were associated with a utility increment of +0.1112 . Additional bleeds and more frequent infusions were associated with lower utility values $(-0.0027$ per bleed and -0.0003 per infusion, respectively).

Conclusion: Subcutaneous prophylaxis is associated with higher utility values compared to intravenous prophylactic and on-demand treatment, while increased bleeds and infusions are associated with reduced utility.

\section{Background}

Hemophilia is a rare congenital disorder that affect predominantly males, and is caused by a mutation of clotting factor genes on the X chromosome (X-linked) that result in a deficiency of factor VIII (FVIII) or -IX (FIX) in hemophilia A or B, respectively. ${ }^{1}$ Globally, 173,711 patients with hemophilia A were identified in 2018, and 3,018 were from Canada. ${ }^{2}$ Bleeding is the main symptom of hemophilia and it occurs after trauma or surgery (including minor/trivial injury), with the severity correlated with the degree of clotting factor deficiency. ${ }^{1}$ Bleeding can occur in muscles, joints, or soft tissue, and in life-threatening cases in the neck, throat, chest, gastrointestinal system, or intracranially. ${ }^{1}$ The main treatment goal is to prevent or treat bleeding; treatment of bleeds is generally via on-demand administration of specific factor concentrate to compensate for the deficient clotting factor, and historically prevention has included prophylaxis regimens of these factor-replacement therapies, ${ }^{1}$ with non-replacement factors having more recently become available. ${ }^{3}$ Other treatment goals are to prevent joint and muscle damage, prevent inhibitor development, prevent transmission of infections from blood products, and improve healthrelated quality of life (HRQoL). ${ }^{1}$ 
Until recently, prophylactic and on-demand treatments have consisted of intravenous exogenous FVIII replacement therapy with recombinant FVIII products or plasma-derived FVIII concentrates. ${ }^{1}$ Historically hemophilia was primarily treated only when bleeding occurred (on-demand); over time the treatment paradigm shifted to prophylaxis with evidence that joint function is better preserved in patients with FVIII levels above $1 \%\left(>1 \mathrm{IU} \mathrm{dL}{ }^{-1}\right) .{ }^{1}$ Based on high quality evidence of the superiority of prophylactic treatment over on-demand treatment, it has become standard of care in Canada for patients with severe hemophilia. ${ }^{1}$ Exogenous FVIII has a short half-life and patients need multiple prophylactic infusions each week in order to maintain adequate trough levels. ${ }^{4,5}$

Although exogenous FVIII concentrate is an effective treatment, one possible serious complication is the development of FVIII inhibitors. ${ }^{1}$ Inhibitors are immunoglobulin $\mathrm{G}$ antibodies that inactivate both exogenous and endogenous FVIII, making FVIII replacement treatment ineffective, at high titres. ${ }^{1,5}$ Approximately $5-10 \%$ of patients with mild to moderate hemophilia $A$, and $20-30 \%$ of patients with severe hemophilia A, develop inhibitors. ${ }^{1}$ Inhibitors can be eradicated with immune tolerance induction (ITI), and bleeding controlled with bypassing agents in patients with inhibitors. ${ }^{6} \mathrm{ITI}$ is not always effective and bleeding control with bypassing agents is often suboptimal compared to FVIII replacement therapy. ${ }^{1}$, 7, 8 Treatment with FVIII at much higher doses, or continuous infusions at "ultra-high doses", may be needed in patients with high levels of inhibitors ( $\geq 5$ Bethesda units) in order to induce immune tolerance. ${ }^{1}$ Therefore, treatments with a lower burden, increased efficacy, and resistance to the development of inhibitors are needed. ${ }^{4,5}$

Emicizumab is a monoclonal antibody that restores the natural function of activated FVIII by bridging activated factor IX and factor $X$ in hemophilia A patients to allow for effective hemostasis. ${ }^{4,}$, 10 Emicizumab, administered subcutaneously, has been shown to be effective in reducing bleeding events in patients with hemophilia A with inhibitors in the HAVEN 1 and 2 trials, ${ }^{11,12}$ as well as in patients with hemophilia A without inhibitors in the HAVEN 3 and 4 trials. ${ }^{4,13}$ Across the clinical trial program, clinical benefits of emicizumab have been observed for weekly, once every two weeks (Q2W), and once every four weeks (Q4W) dosing schedules (with Q4W dosing recommended only for adults and/or adolescents > $40 \mathrm{~kg}$ [in the Canadian label]). ${ }^{14}$

The HRQoL of patients with hemophilia is negatively affected by both the disease and treatment. ${ }^{15}$ Recurrent bleeding and resulting complications such as joint and muscle damage, and pain and disability, can significantly affect patients' HRQoL. ${ }^{15}$ Treatment-related factors include the need for frequent infusions due to the half-life of available therapies, and specific infusion-related problems such as difficulty with accessing veins, the time required to administer treatment, and development of inhibitors - all of which can have a negative effect on treatment adherence, lifestyle, and HRQoL. ${ }^{16}$ Quality-adjusted life years (QALYS), a measure of health that quantifies both quality and length of life, are used to assess the effectiveness of health technologies. Quality of life is quantified in utility values, on a scale where 0 is equivalent to death and 1 represents perfect health, where states worse than death (less 
than 0) may exist. ${ }^{17}$ Alternately, visual analogue scale (VAS) measures of HRQoL are obtained by asking participants where they would rate their overall health on a 'scale' of $0-100$, with 0 being equivalent to death and 100 perfect health. Based on values from the 2013-2014 Canadian Community Health Survey, the mean and median self-reported utility scores for Canadians were estimated using the Health Utilities Index Mark 3 (HUI3) at 0.86 and 0.93 , respectively. ${ }^{18}$ A cross-sectional study of 101 Canadian patients with hemophilia found that they have increased morbidity compared to the general population [(HUI3 of $0.71 \pm 0.29$ for those with hemophilia vs. 0.91 for the general population), and that the burden of morbidity was correlated with disease severity [mean HUI3 of $0.79 \pm 0.30$ for mild hemophilia vs. $0.71 \pm$ 0.23 for severe hemophilia vs. $0.57 \pm 0.32$ for severe hemophilia with human immunodeficiency virus (HIV)] ${ }^{19}$ In addition to hemophilia patients, having a family member with hemophilia inevitably increases the burden on the caregivers and the family, especially when a child is affected. ${ }^{20,21}$ The emotional and practical aspects of caregiver burden are especially high when the patient requires frequent infusions of hemophilia treatment. Worse social, physical, emotional, financial, and lifestyle impact were associated with more infusions per week in a large study of hemophilia caregivers. ${ }^{22}$

A study from six countries, including Canada, reported that Canadian patients with severe hemophilia (n $=40$ ) had the second highest EuroQuol-5D (EQ-5D) utility scores $(0.791) \cdot{ }^{23}$ In comparison, the mean the EQ-5D (using an European value set) was 0.925 for the general population in Alberta, Canada. ${ }^{24}$ In another study, Canadian adults with hemophilia report better Standard Gamble utilities than the general population ( 0.94 to 0.90$).{ }^{15}$ One possible reason for this discrepancy is the "disability paradox" where patients have adapted to their specific situation to such an extent that they report similar or higher HRQoL utilities than the general population. ${ }^{25,26}$ Another explanation may be the instrument that was used. Of the primary techniques, the Standard Gamble generates the highest utility values, ${ }^{25}$ and may overestimate utility scores while underestimating the impact of worse health states. ${ }^{6}$ These and other issues present challenges for economic evaluations of treatments.

Within an economic evaluation, health state utilities can be informed by either patient-reported values, or by HRQoL measures evaluated by members of the general population to reflect community values. ${ }^{6,27}$ In the United States and United Kingdom there is a preference for using utilities derived from the general population/societal perspective rather than utilities from patients to assign a value to a specific health state. ${ }^{6}$ To date, cost utility analysis (CUA)s that compare on-demand treatment with prophylactic treatment for hemophilia have reported inconsistent results, with uncertainty in health state utilities being an important source of variability. ${ }^{28}$

Factors that positively influenced willingness to pay from a patient-perspective include increased effectiveness of treatments, reduced adverse events, and reduced dosing frequency. ${ }^{29}$ Patients generally prefer prophylactic to on-demand treatment, likely due to improved clinical outcomes with prophylactic treatment. ${ }^{29}$ However, the impact of frequent intravenous infusions and the type of treatment on HRQoL has not been explored adequately. ${ }^{6}$ Recently, subcutaneous treatments have become available for 
hemophilia A, and while currently emicizumab is the only available subcutaneous therapy, other pipeline therapies are likely to become available in the future. There are challenges with understanding health state utilities for subcutaneous treatments because the impact of subcutaneous administration compared to intravenous administration has not been previously explored in hemophilia patients. However, there are studies in patients with other chronic conditions that found a preference for subcutaneous administration compared to intravenous administration. ${ }^{30-32}$ Current intravenous hemophilia treatments can be time-consuming, challenging, complex, and painful to administer, and can lead to difficulty in coordinating schedules (including work schedules) and maintaining a routine, cause anxiety, trauma, exhaustion, social stigma, and impact personal relationships and career opportunities. ${ }^{15}$ Participants in a Canadian survey study reported that delivery via an alternate route of administration (subcutaneous or oral), and treatments with longer-lasting effects in order to reduce the frequency of administration, would be substantial improvements over the current standard of care. ${ }^{33}$

\section{Methods}

The aim of this study was to estimate utility values associated with hemophilia A patients' health and treatments [on demand, infusion prophylaxis, and subcutaneous prophylaxis] amongst members of the Canadian general public. Results of this analysis can be used to populate Canadian cost-utility analysis of subcutaneous therapy vs. intravenous prophylaxis and on-demand therapy, respectively.

A de novo utilities study was conducted in a Canadian general population sample to compare HRQoL across treatment states, including the HRQoL implications of bleeds, treatment modality, and frequency of infusions. A convenience sample was recruited via market research panels and snowball sampling, with demographics intended to approximate the Canadian population. A vignette-based time trade-off (TTO) utilities elicitation was undertaken to compare population preferences for subcutaneous treatment compared to intravenous prophylaxis and on-demand treatment. The general population study conducted here allows for consideration of a Canadian societal perspective. Hemophilia A health states were developed based on clinical investigator experience (two clinicians) with treating this population, and indepth, semi-structured qualitative interviews with eight Canadian adult caregivers of children with moderate to severe hemophilia A and two Canadian adults with moderate to severe hemophilia A. The objective was to understand the specific disease and treatment burdens that patients with hemophilia $A$ face, as well as their preferences for treatment options. Transcripts were analyzed with Hyper Research software and a content and thematic analysis was conducted. ${ }^{34}$

\section{Clinical Inputs:}

Clinical inputs into health states, where available, were sourced from the HAVEN 3 and HAVEN 4 trials and published literature. HAVEN 3 was a randomized, multicenter, open-label, Phase III clinical study in 152 adult and adolescent males (age $\geq 12$ years) with severe hemophilia A without FVIII inhibitors who previously received either episodic (on demand) or prophylactic treatment with FVIII. ${ }^{4,35}$ Two different 
treatment regimens were evaluated relative to episodic treatment with FVIII: prophylactic treatment with emicizumab at doses of $1.5 \mathrm{mg} / \mathrm{kg}$ weekly (QW) or $3 \mathrm{mg} / \mathrm{kg}$ Q2W. Patients who received episodic FVIII prior to enrolment were randomized to one of the two emicizumab maintenance regimens (Arm A: $1.5 \mathrm{mg} / \mathrm{kg}$ QW, Arm B: $3 \mathrm{mg} / \mathrm{kg}$ Q2W, or to Arm C: no prophylaxis). In addition to the randomized component, HAVEN 3 included a non-interventional study (NIS) in which patient characteristics and clinical outcomes such as bleed rates were retrospectively extracted from charts of patients receiving FVIII prophylaxis prior to study initiation. ${ }^{4}$ HAVEN 4 is an open-label, multicenter, Phase III clinical study in adults and adolescents ( $\geq 12$ years) with severe congenital hemophilia A or hemophilia A with FVIII inhibitors who were previously treated with FVIII concentrates or recombinant factor VII ( $\mathrm{FFVIIa),} \mathrm{or} \mathrm{were}$ willing to switch to $\mathrm{rFVIla}$ to treat breakthrough bleeds. ${ }^{35}$ Participants received a loading dose of subcutaneous emicizumab $3 \mathrm{mg} / \mathrm{kg}$ Q4W (monthly), followed by $6 \mathrm{mg} / \mathrm{kg}$ Q4W for 24 weeks as prophylaxis. $^{35}$

\section{Interventions:}

Health states were designed to describe prophylactic subcutaneous treatment, prophylactic intravenous infusion, and on-demand interventions.

\section{Outcomes:}

Overall, 6 health states were evaluated (Fig. 1):

- Health State A: On-demand therapy, with 36 bleeds per year (3 per month)

- Health State B: intravenous prophylaxis with 2-3 infusions per week and 4-5 bleeds per year

- Health State C: intravenous prophylaxis with 2-3 infusions per week and 10 bleeds per year

- Health State D: intravenous prophylaxis with daily infusions and 4-5 bleeds per year

- Health State E: Subcutaneous prophylaxis with weekly injections and 1-2 bleeds per year

- Health State F: Subcutaneous prophylaxis with monthly injections and 1-2 bleeds per year

Health states A, B, and E correspond closely to the HAVEN 3 results, while Health states C, D, and F were included to assess the impact on number of bleeds, frequency of infusions, and frequency of injections on reported utilities. All health states and vignettes were drafted in two distinct versions: one based on the experience of a child with hemophilia, and one based on the experience of an adult with hemophilia, and respondents were randomly allocated to one of the two versions. TTO utilities were elicited via a "ping-

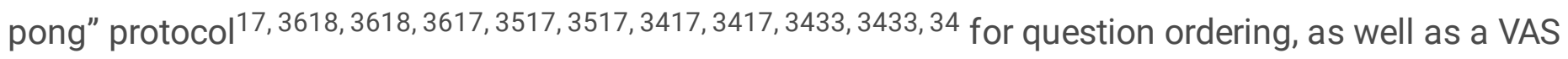
rating. ${ }^{17,36}$ Interviews were conducted according to standard TTO protocols by trained interviewers via videoconference and data were entered directly into a secured server. ${ }^{17,36}$ Ethical approval was obtained for both the qualitative interview and quantitative survey components of the study.

\section{Analyses:}


Three key analyses were undertaken for both TTO utilities and VAS scores: (1) a descriptive analysis of mean and $95 \%$ confidence interval $(\mathrm{Cl})$ by health state, $(2)$ a mixed-effects (random-intercept by patient) analysis of utility values as predicted by subcutaneous vs. infusion-based therapies and number of bleeds, and (3) for prophylactic regimens, an analysis of utilities by frequency of infusions or injections, to understand the impact and potential disutility of infusions and injections. In the first random-intercept model, adjustments were made for subcutaneous vs. intravenous treatment modalities and the number of bleeds per year. Note that in this regression analysis, covariates for age, sex, and adult vs. child survey were tested, but none of these parameters were found to be statistically significant and thus were not included in the final models and associated results. In the second model, only prophylactic regimens were considered, and utilities and VAS scores were assessed based on frequency of infusions and injections, respectively (i.e. by comparing results of Health States B vs. D and Health States E vs. F). Additionally, a sensitivity analysis of a hypothetical health state was evaluated where annual bleed rate (ABR) was reduced from 4.5 to 1.5 to reflect patients that are well controlled with prophylaxis. Weekly subcutaneous prophylaxis was compared to daily intravenous prophylaxis for this health state.

\section{Results}

The sample consisted of 82 adult respondents, of whom $59 \%$ were women and $41 \%$ were men. Fifty-four percent answered the adult version of the survey, while the remaining $46 \%$ answered the pediatric version. Crude mean and 95\% $\mathrm{Cl}$ results are reported in Table 1 and can be visualized in Fig. 2a. Utilities were highest for subcutaneous health states and lowest for on-demand therapy. Health states A, B, and E, which correspond to base case model health states, were associated with utilities of 0.70 (on-demand), 0.81 (intravenous prophylaxis), and 0.90 (subcutaneous prophylaxis). Additional bleeds and more frequent infusions and injections were associated with lower utility values, although the values for weekly vs. monthly subcutaneous injections were close ( 0.90 vs. 0.91 , with highly overlapping Cls). The VAS results followed similar trends, although with relatively lower results on the 0-100 scale compared to TTO utilities on the $0-1$ scale (Table 1 ; Fig. 2 b). 
Table 1

Mean and $95 \%$ confidence intervals (CI) for utility and visual analogue scale (VAS) results

\section{Mean $(95 \% \mathrm{Cl})$}

\begin{tabular}{|lll|}
\hline & Utility & VAS \\
\hline A. On demand; ABR = 36 & $0.70(0.65-$ & $56.2(52.9-$ \\
& $0.76)$ & $59.5)$ \\
\hline B. Intravenous prophylaxis; 2-3 infusions per week; ABR =4- & $0.81(0.78-$ & $71.1(68.3-$ \\
5 & $0.85)$ & $73.9)$ \\
\hline C. Intravenous prophylaxis; 2-3 infusions per week; ABR = 10 & $0.78(0.73-$ & $67.1(64.1-$ \\
& $0.82)$ & $70.0)$ \\
\hline D. Intravenous prophylaxis w/ daily infusions; ABR = 4-5 & $0.74(0.69-$ & $63.7(59.8-$ \\
& $0.80)$ & $67.6)$ \\
\hline E. Subcutaneous prophylaxis Q1W; ABR = 1-2 & $0.90(0.87-$ & $81.6(78.9-$ \\
& $0.93)$ & $84.2)$ \\
\hline F. Subcutaneous prophylaxis Q4W; ABR = 1-2 (ABR = 1-2) & $0.91(0.88-$ & $84.5(82.0-87.1)$ \\
& $0.94)$ & \\
\hline ABR = annual bleed rate; Cl = confidence interval; Q1W = once weekly injection; Q4W = once monthly \\
injection; VAS = visual analogue scale
\end{tabular}

In the utilities analysis, subcutaneous treatment was associated with a utility increment of +0.1112 , and each bleed per year a decrement of -0.0027 (Table 2). Thus, while respondents indicated a preference for health states with fewer bleeds, the preference for a subcutaneous vs. intravenous treatment was stronger. For health state $E$, the regression-predicted utility is 0.903 . Health state $B$ is associated with a regression-predicted utility of 0.784 , reflecting lower utility than health state $E$ related to intravenous vs. subcutaneous route of administration, and increased bleeds (4.5 vs. 1.5). However, in a hypothetical health state where the number of bleeds is decreased from 4.5 to 1.5 but intravenous route of administration, the resulting predicted utility is 0.792 - higher than that of health state B, but notably lower than that of health state $\mathrm{E}$. 
Table 2

Regression coefficients in random-intercept analysis of utilities and visual analogue scale (VAS) results

\begin{tabular}{|lllll|}
\hline & Utilities & & VAS & \\
\hline & Coefficient & p-value & Coefficient & p-value \\
\hline Intercept & 0.7966 & $<0.001$ & 69.9 & $<0.001$ \\
\hline Bleeds per year & -0.0027 & $<0.001$ & -0.4 & $<0.001$ \\
\hline Subcutaneous treatment* & 0.1112 & $<0.001$ & 13.6 & $<0.001$ \\
\hline VAS = visual analogue scale & & & \\
\hline *vs. reference category of intravenous infusions & & \\
\hline
\end{tabular}

In the intravenous prophylaxis model, each annual intravenous infusion was associated with a statistically significant disutility of -0.0003 , while each annual subcutaneous injection was associated with an estimated disutility of -0.0002 , although this latter difference was not found to be statistically significant due to wider variability in patient responses. This is generally consistent with the results reported in Table 1 and Fig. 2a, in which similar utility estimates were found for weekly vs. monthly subcutaneous injection regimens.

\section{Discussion}

This paper reports utility values from a Canadian societal perspective for different health and treatment states, including the HRQoL implications of bleeds, treatment modality [prophylactic subcutaneous treatment vs. intravenous infusion prophylaxis and on-demand therapy], and frequency of infusions. Members of the Canadian population preferred subcutaneous health states compared to intravenous infusions, and to a lesser extent health state utilities also reflected a desire to avoid additional bleeding episodes and infusions. Therefore, evidence suggests that the benefit of subcutaneous administration was more important than the number of bleeding episodes within the likely range of bleeding episodes to occur with prophylactic regimens. VAS results were 56.2 for on-demand treatment, 71.1 for intravenous infusion prophylaxis and 81.6 for subcutaneous prophylaxis, while utilities were 0.70 for on-demand treatment, 0.81 for intravenous prophylaxis, and 0.90 for subcutaneous prophylaxis. This aligns with a study in 22 hemophilia patients with inhibitors compared prophylaxis with on-demand treatment and found that prophylaxis resulted in a reduction in bleeding frequency which improved HRQoL. ${ }^{37}$ Additionally, prophylactic treatment decreased absenteeism and pain, and increased mobility and median VAS and TTO scores (from 66-73, $p=0.048$; and from 0.62-0.76, $p=0.054$, respectively). ${ }^{37}$ Other studies had similar findings. ${ }^{38-40} \mathrm{~A}$ study of 401 boys with hemophilia from nine countries, including 168 from Canada (82.7\% with hemophilia A) assessed the effect of prophylaxis and disease severity on HRQOL and found that prophylactic treatment significantly increased HRQoL compared to on-demand treatment (mean Canadian Hemophilia Outcomes-Kids' Life Assessment Tool (CHO-KLAT) score (SD) of 74.5 (12.7) 
vs. 71.7 (13.1), respectively), whereas increased disease severity was associated with significantly lower HRQoL compared to mild or moderate disease. ${ }^{41}$ Mean CHO-KLAT score (SD) of Canadian boys was 75.0 (13.0). ${ }^{41}$ A CUA comparing primary prophylactic treatment with on-demand treatment in Canadian boys with severe hemophilia A, found that HRQoL and clinical outcomes are substantially improved with prophylaxis compared to on-demand treatment, but it comes at a substantial incremental cost per QALY. ${ }^{42}$ In a prior study it was not found that prophylactic treatment improved HRQoL, but there were several limitations noted, including not taking into account the impact of frequent intravenous infusions on HRQoL. ${ }^{43}$

Although a societal perspective is important, patient perspective is also important, since treatment improvements may have positive impacts on treatment adherence, employment (economic), social, and health aspects. ${ }^{33}$ The Institute for Clinical and Economic Review (ICER; United States) recently released a report assessing the clinical effectiveness and value of current hemophilia A therapies indicating that, compared to factor VIII prophylaxis, subcutaneous therapy reduces patient and caregiver burden and may improve patient outcomes. ${ }^{44} \mathrm{~A}$ qualitative study of 12 Canadian patients with hemophilia $\mathrm{A}$ and eight caregivers found several challenges with current treatments which negatively affect participants' quality of life, including their physical, social, and psychological well-being. ${ }^{33}$ Based on the findings of studies in patients with other conditions, treatments with a different route of administration may address some of these problems. A systematic review compared patient-preference for intravenous versus subcutaneous administration across a diverse patient population and treatments, and found that patients clearly preferred subcutaneous administration over intravenous administration, primarily due to factors related to time and convenience. ${ }^{32}$ This may have implications for HRQoL and adherence to treatment. ${ }^{32}$ Another systematic review of patient preferences regarding treatment for rheumatoid arthritis found that sociodemographic characteristics influenced patient preferences, but that the benefit of the treatment was more important than the route of administration, adverse events, or costs. ${ }^{30}$ However, patients often preferred subcutaneous treatment over intravenous treatment. ${ }^{30} \mathrm{~A}$ systematic review to determine the burden of treatment in patients with primary immunodeficiency disorders found that the burden of treatment was generally low overall and patients were satisfied with both intravenous and subcutaneous immunoglobulin treatment, however once patients switched from intravenous to subcutaneous, subcutaneous treatment was preferred. ${ }^{31}$ These studies were not conducted specifically in patients with hemophilia, and an interesting question would be whether patients with hemophilia A who are currently being treated with intravenous FVIII would still rank their HRQoL with current treatment as high once they have experienced treatment with a different treatment modality that is administered subcutaneously.

\section{Limitations}

To our knowledge, this is the first study to compare preferences for intravenous vs. subcutaneous prophylaxis in hemophilia. This study used a vignette-based TTO method in a general population sample. According to a study that compared the agreement between EQ-5D-3L and vignette-based TTO, vignettebased TTO methods tend to generate lower utility scores than the EQ-5D, and disagreement between the 
two methods are increased with chronic diseases compared to acute disease. ${ }^{45}$ This can lead to systematic differences between resulting cost-effectiveness calculations across the two methods; the aim with TTO health states is to develop them to be sufficiently nuanced as to capture the key features of living with the disease, but not to the point where complexity and/or subjectivity create challenges for respondents. ${ }^{45}$ Of note, however, the impact of TTO vs. EQ-5D utilities is not anticipated to affect different health states differentially. Although the absolute values of all utilities may have been higher in an EQ-5D study, there is no a priori expectation that the incremental difference between intravenous and subcutaneous health states would have been different.

\section{Next Steps}

As real-world data accrue from hemophilia A patients with and without inhibitors, having experience with both intravenous and subcutaneous regimens, a companion HRQoL study from the patient perspective would provide valuable insights to the general population results described here.

\section{Conclusions}

Subcutaneous prophylaxis is associated with higher utility values compared to intravenous prophylaxis and on-demand treatment, while increased bleeds and infusions resulted in lower utility values.

\section{Abbreviations}

$\mathrm{ABR}=$ annual bleed rate; $\mathrm{CHO}-\mathrm{KLAT}=$ Canadian Hemophilia Outcomes-Kids' Life Assessment Tool; $\mathrm{Cl}=$ confidence interval; CUA = cost utility analysis; EQ-5D = EuroQuol-5D; FVIII = factor VIII; FVIX = factor IX; $\mathrm{HIV}=$ human immunodeficiency virus; $\mathrm{HRQoL}=$ health-related quality of life; $\mathrm{HUI} 3=$ Health Utilities Index Mark 3; ITI = immune tolerance induction; NIS = non-interventional study; Q1W = once weekly; Q2W = once bi-weekly (every 2 weeks); Q4W = once monthly (every 4 weeks); QALY = quality-adjusted life years; $\mathrm{QW}=$ once weekly; rFVIla = recombinant factor VIl; TTO = time trade-off; VAS = visual analogue scale

\section{Declarations}

\section{Ethics approval and consent to participate}

This study was approved by Veritas Institutional Review Board on April 24, 2020 (IRB Tracking Number: 16556-17:21:0124-04-2020).

\section{Consent for publication:}

The publication of study results was not contingent on the sponsor's approval or censorship of the manuscript.

\section{Availability of data and materials}


Anonymized data from this study can be provided upon request.

\section{Competing interests}

Not applicable

\section{Funding}

This work was sponsored by Hoffmann-La Roche Ltd.

\section{Authors' contributions}

SO and DD contributed to the study design, LE and RB conducted hemophilia A patient recruitment and qualitative interviews, KJ, ATM, SO, and DD designed the TTO utilities and VAS study protocol, JK and ATM conducted the online interviews, JMS and RJK provided expert clinical experience, $\mathrm{KJ}$ analyzed the data, KJ, ATM, SO , and DD were main contributors in writing the manuscript. All authors read and approved the final manuscript.

\section{Acknowledgements}

We would like to acknowledge Sarah Clifford's and Inka Karppinen's contributions to the development of this manuscript.

\section{References}

1. Srivastava A, Brewer A, Mauser-Bunschoten E and et al. WFH Guidelines for the management of hemophilia. Haemophilia. 2013; 19: e1-e47.

2. World Federation of Hemophilia. Report on the annual global survey 2018. 20 ed. Montréal, Québec2019, p. 88.

3. Weyand AC and Pipe SW. New therapies for hemophilia. Blood. 2019; 133: 389-98.

4. Mahlangu J, Oldenburg J, Paz-Priel I, et al. Emicizumab Prophylaxis in Patients Who Have Hemophilia A without Inhibitors. New England Journal of Medicine. 2018; 379: 811-22.

5. Peyvandi F, Garagiola I and Seregni S. Future of Coagulation Factor Replacement Therapy. J Thromb Haemost. 2013; 11: 84-98.

6. Grosse S, Chaugule S and Hay J. Estimates of utility weights in hemophilia: implications for cost utility analysis of clotting factor prophylaxis. Expert Rev Pharmacoecon Outcomes Res. 2015; 15: 267-83.

7. Rocino A, Franchini M and Coppola A. Treatment and Prevention of Bleeds in Haemophilia Patients with Inhibitors to Factor VIII/IX. Journal of Clinical Medicine. 2017; 6: 1-18.

8. Hay $C$ and DiMichele D. The principal results of the International Immune Tolerance Study: a randomized dose comparison. Blood. 2012; 119: 1335-44. 
9. Institute for Clinical and Economic Review. Emicizumab for Hemophilia A with Inhibitors: Effectiveness and Value. CEPAC, 2018, p. 1-162.

10. Roche Canada. Positive phase III results for Roche's Hemlibra for haemophilia A without factor VIII inhibitors published in New England Journal of Medicine. 2018.

11. Oldenburg J, Mahlangu J, Kim B and et al. Emicizumab prophylaxis in hemophilia A with inhibitors. N Engl J Med. 2017; 377: 809-19.

12. Young G, Sidonio R, Liesner R and et al. HAVEN 2 updated analysis: multicenter, open-label, phase 3 study to evaluate efficacy, safety and pharmacokinetics of subcutaneous administration of emicizumab prophylaxis in pediatric patients with hemophilia A with inhibitors. The Annual meeting of the American Society of Hematology. Atlanta, GA2017.

13. Pipe S, Shima M, Lehle M and et al. Efficacy, safety, and pharmacokinetics of emicizumab prophylaxis given every 4 weeks in people with haemophilia A (HAVEN 4): a multicentre, open-label, non-randomised phase 3 study. The Lancet Haematology. 2019; 6: e295-e305.

14. Hoffmann-La Roche Limited. Product Monograph: Pr HEMLIBRA® subcutaneous emicizumab injection. Mississauga, ON: Health Canada, 2019.

15. Naraine V, Risebrough N, Oh P and et al. Health-related quality-of-life treatments for severe haemophilia: utility measurements using the Standard Gamble technique. Haemophilia. 2002; 8: $112-20$.

16. Santagostino E, Lentz S, Busk A and et al. Assessment of the impact of treatment on quality of life of patients with haemophilia $A$ at different ages: insights from two clinical trials on turoctocog alfa. Haemophilia. 2014; 20: 527534.

17. Oppe M, Rand-Hendriksen K, Shah K and et al. EuroQol Protocols for Time Trade-Off Valuation of Health Outcomes. PharmacoEconomics. 2016; 34: 993-1004.

18. Guertin JR, Feeny D and Tarride JE. Age- and sex-specific Canadian utility norms, based on the 20132014 Canadian Community Health Survey. CMAJ : Canadian Medical Association journal = journal de l'Association medicale canadienne. 2018; 190: E155-e61.

19. Barr R, Saleh $M$, Furlong $W$ and et al. Health status and health-related quality of life associated with hemophilia. American Journal of Hematology. 2002; 71: 152-60.

20. DeKoven $M$, Karkare S, Lee W and et al. Impact of haemophilia with inhibitors on caregiver burden in the United States. Haemophilia. Haemophilia. 2014; 20: 822-30.

21. Khair K and Von Mackensen S. Caregiver burden in haemophilia: results from a single UK centre. The Journal of Haemophilia Practice. The Journal of Haemophilia Practice. 2017; 4: 40-8.

22. Schwartz C, Powell V, Su J and et al. The impact of extended half-life versus conventional factor product on hemophilia caregiver burden. Quality of Life Research. 2018; 27: 1335-45.

23. Noone D, O'Mahony B, van Dijk J and et al. A survey of the outcome of prophylaxis, on-demand treatment or combined treatment in 18-35-year old men with severe haemophilia in six countries. Haemophilia. 2013; 19: 44-50. 
24. Janssen B and Szende A. Chapter 3: Population Norms for the EQ-5D. In: Szende A, Janssen B and Cabases J, (eds.). Self-reported Population Health: An International Perspective based on EQ-5D [Internet]. Dordrecht, NL: Springer, 2014.

25. Farrugia A, Mahony B and Cassar J. Health technology assessment and haemophilia. Haemophilia. 2012; 18: 152-7.

26. Ubel P, Loewenstein G, Schwarz $N$ and et al. Misimagining the unimaginable: the disability paradox and health care decision making. Health Pschol. 2005; 24: S57-S62.

27. Rawlins $M$ and Culyer A. National Institute for Clinical Excellence and its value judgments. BMJ. 2004; 329: 224-7.

28. Thorat T, Neumann P and Chambers J. Hemophilia Burden of Disease: A Systematic Review of the Cost-Utility Literature for Hemophilia. J Manag Care Spec Pharm. 2018; 24.

29. Chaugule S, Hay J and Young G. Understanding patient preferences and willingness to pay for hemophilia therapies. Patient Preference and Adherence. 2015; 9: 1623-30.

30. Durand C, Eldoma M, Marshall D and et al. Patient Preferences for Disease-modifying Antirheumatic Drug Treatment in Rheumatoid Arthritis: A Systematic Review. J Rheumatol. 2020; 47: 176-87.

31. Jones G, Vogt K, Chambers D and et al. What Is the Burden of Immunoglobulin Replacement Therapy in Adult Patients With Primary Immunodeficiencies? A Systematic Review. Front Immunol. 2018; 9: 1308.

32. Stoner K, Harder H, Fallowfield L and et al. Intravenous versus subcutaneous drug administration. Which do patients prefer? A systematic review. The Patient. 2015; 8: 145-53.

33. Wiley R, Khoury C, Snihur A and et al. From the voices of people with haemophilia A and their caregivers: Challenges with current treatment, their impact on quality of life and desired improvements in future therapies. Haemophilia. 2018; 25: 433-40.

34. Joffe $\mathrm{H}$ and Yardley L. Content and thematic analysis. Research methods for clinical and health psychology. London: Sage, 2004, p. 56-68.

35. ClinicalTrials.gov. A Study to Evaluate the Efficacy, Safety, Pharmacokinetics, and Pharmacodynamics of Emicizumab Given Every 4 Weeks in Participants With Hemophilia A (HAVEN 4) NCT03020160. 2020.

36. Shen J, Hill S, Mott D and et al. Conducting a Time Trade-Off Study Alongside a Clinical Trial: A Case Study and Recommendations. PharmacoEconomics. 2019; 3: 5-20.

37. Hoots W, Ebbesen L, Konkle B and et al. Secondary prophylaxis with recombinant activated factor VII improves health-related quality of life of haemophilia patients with inhibitors. Haemophilia. 2008; 14: 466-75.

38. Noone D, O'Mahony B and Prihodova L. A survey of the outcome of prophylaxis, on-demand or combined treatment in 20-35 year old men with severe haemophilia in four European countries (letter). Haemophilia. 2011; 17: E842-E3. 
39. Oladapo A, Epstein J, Williams E and et al. Health-related quality of life assessment in haemophilia patients on prophylaxis therapy: a systematic review of results from prospective clinical trials. Haemophilia. 2015; 21: e344-e58.

40. Stasyshyn O, Antunes S, Mamanov V and et al. Prophylaxis with anti-inhibitor coagulant complex improves health-related quality of life in haemophilia patients with inhibitors: results from FEIBA NF Prophylaxis Study. Haemophilia. 2014; 20: 644-50.

41. Usuba K, Price V, Blanchette $V$ and et al. Impact of prophylaxis on health-related quality of life of boys with hemophilia: An analysis of pooled data from 9 countries. Res Pract Thromb Haemost. 2019; 3: 397-404.

42. Risebrough N, Oh P, Blanchette V and et al. Cost-utility analysis of Canadian tailored prophylaxis, primary prophylaxis and on-demand therapy in young children with severe haemophilia A. Haemophilia. 2008; 14: 743-52.

43. Buchbinder $D$ and Ragni $V$. What is the role of prophylaxis in the improvement of health-related quality of life of patients with hemophilia? Hematology Am Soc Hematol Educ Program. 2013: 52-5.

44. Institute for Clinical and Economic Review. Valoctocogene Roxaparvovec and Emicizumab for Hemophilia A without Inhibitors: Effectiveness and Value: Final Report. Institute for Clinical and Economic Review. 2020.

45. Briggs $A$, Belozeroff $\vee$ and Feeny D. Comparison of health state utility estimates from instrumentbased and vignette-based methods: a case study in kidney disease. BMC Res Notes. 2019; 12: 385.

\section{Figures}


Health State A: on-demand therapy, with 36 bleeds per year (3 per month)

Health State B: intravenous prophylaxis with 2-3 infusions per week and 4-5 bleeds per year

Health State C: intravenous prophylaxis with 2-3 infusions per week and 10 bleeds per year

Health State D: intravenous prophylaxis with daily infusions and 4-5 bleeds per year

Health State E: subcutaneous prophylaxis with weekly injections and 1-2 bleeds per year

Health State F: subcutaneous prophylaxis with monthly injections and 1-2 bleeds per year

Figure 1

Model health states to describe prophylactic subcutaneous treatment, prophylactic intravenous infusion, and on-demand interventions 
a.

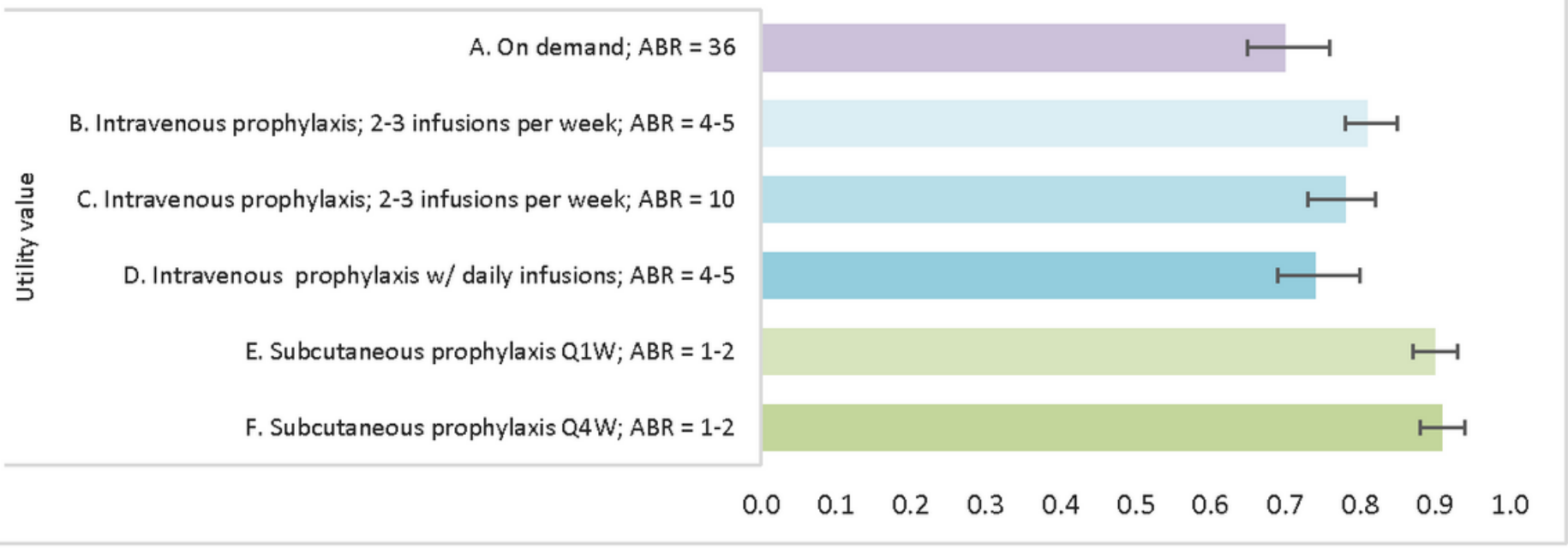

b.

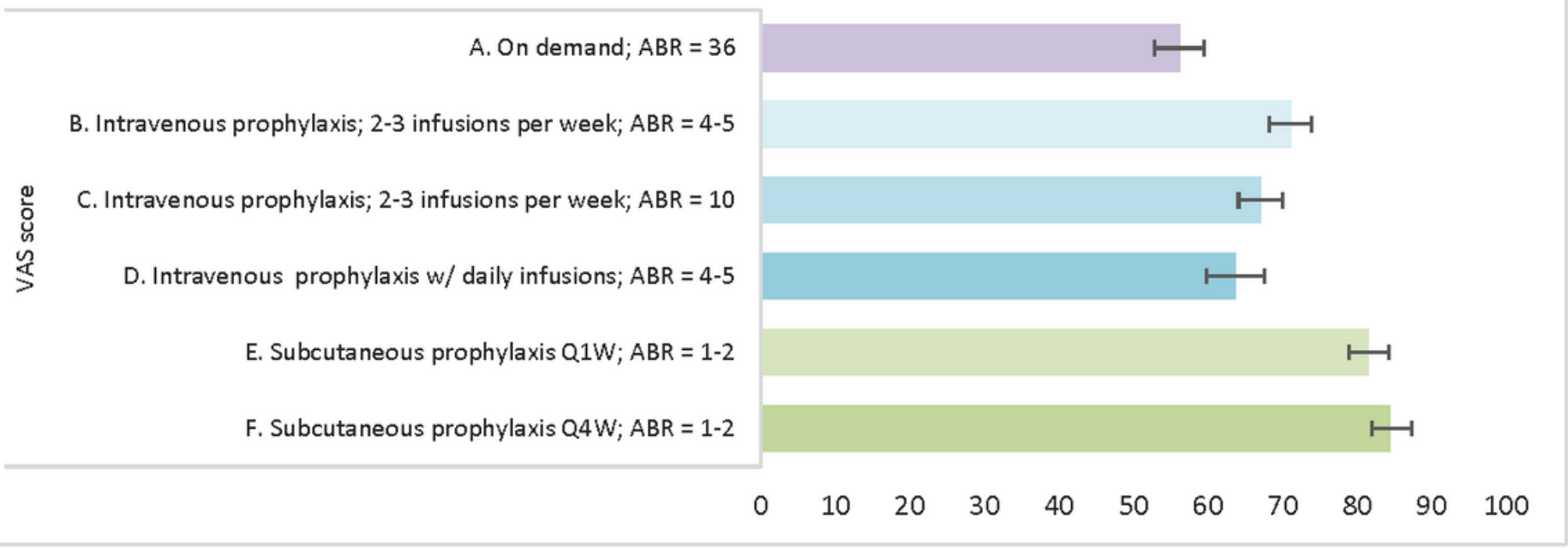

$\mathrm{ABR}=$ annual bleed rate; $\mathrm{CI}=$ confidence interval; $\mathrm{Q} 1 \mathrm{~W}=$ once weekly injection; $\mathrm{Q} 4 \mathrm{~W}=$ once monthly injection; VAS = visual analogue scale

\section{Figure 2}

Mean and 95\% confidence intervals $(\mathrm{Cl})$ for a. utility and b. visual analogue scale (VAS) results $A B R=$ annual bleed rate; $\mathrm{Cl}=$ confidence interval; Q1W = once weekly injection; Q4W = once monthly injection; VAS = visual analogue scale 